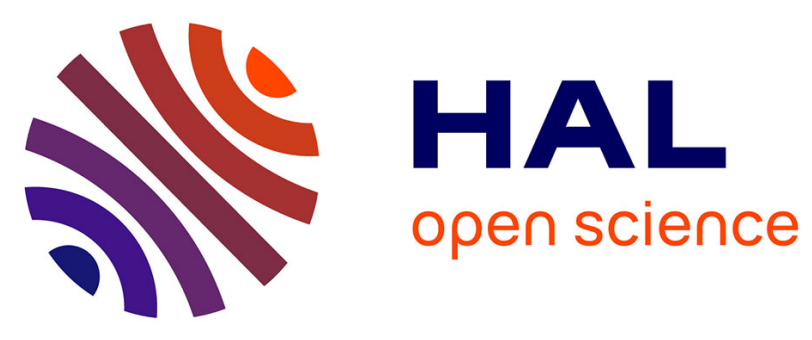

\title{
A graph-based approach to detect spatiotemporal dynamics in satellite image time series
}

Fabio N. Güttler, Dino Ienco, Jordi Nin, Maguelonne Teisseire, Pascal Poncelet

\section{- To cite this version:}

Fabio N. Güttler, Dino Ienco, Jordi Nin, Maguelonne Teisseire, Pascal Poncelet. A graph-based approach to detect spatiotemporal dynamics in satellite image time series. ISPRS Journal of Photogrammetry and Remote Sensing, 2017, 130, pp.92-107. 10.1016/j.isprsjprs.2017.05.013 . lirmm-01541930

\section{HAL Id: lirmm-01541930 \\ https://hal-lirmm.ccsd.cnrs.fr/lirmm-01541930}

Submitted on 19 Jun 2017

HAL is a multi-disciplinary open access archive for the deposit and dissemination of scientific research documents, whether they are published or not. The documents may come from teaching and research institutions in France or abroad, or from public or private research centers.
L'archive ouverte pluridisciplinaire HAL, est destinée au dépôt et à la diffusion de documents scientifiques de niveau recherche, publiés ou non, émanant des établissements d'enseignement et de recherche français ou étrangers, des laboratoires publics ou privés. 
Enhancing the frequency of satellite acquisitions represents a key issue for Earth Observation community nowadays. Repeated observations are crucial for monitoring purposes, particularly when intra-annual process should be taken into account. Time series of images constitute a valuable source of information in these cases. The goal of this paper is to propose a new methodological framework to automatically detect and extract spatiotemporal information from satellite image time series (SITS).Existing methods dealing with such kind of data are usually classification-oriented and cannot provide information about evolutions and temporal behaviors. In this paper we propose a graph-based strategy that combines object-based image analysis (OBIA) with data mining techniques. Image objects computed at each individual timestamp are connected across the time series and generates a set of evolution graphs. Each evolution graph is associated to a particular area within the study site and stores information about its temporal evolution. Such information can be deeply explored at the evolution graph scale or used to compare the graphs and supply a general picture at the study site scale. We validated our framework on two study sites located in the South of France and involving different types of natural, semi-natural and agricultural areas. The results obtained from a Landsat SITS support the quality of the methodological approach and illustrate how the framework can be employed to extract and characterize spatiotemporal dynamics.

* corresponding author

Email addresses: fabioguttler@gmail.com (Fabio Guttler ), dino.ienco@teledetection.fr (Dino Ienco), nin@ac.upc.edu (Jordi Nin), maguelonne.teisseire@teledetection.fr (Maguelonne Teisseire), pascal.poncelet@lirmm.fr (Pascal Poncelet)

Preprint submitted to ISPRS J. Photogramm. Remote Sens. 


\section{Introduction}

Nowadays, satellite image time series (SITS) is a powerful source of information for monitoring purposes. Repeated satellite observations allow to follow the evolution (e.g. growing season, land-cover modifications) of a given area over the time in a systematic way. When repeatability and homogeneity of satellite observations are guaranteed it becomes possible to detect spatiotemporal evolutions and deduce their related dynamics (Bonn, 1996). However, the interpretation and the cross-comparison of several satellite images quickly become challenging.

Advanced methods used to process multitemporal optical imagery are related to trajectory analysis. In this context, high-temporal frequency SITS from coarse to moderate sensors, such as MODIS, are used to model temporal signatures and detect anomalies or trends (Lunetta et al., 2006; Verbesselt et al., 2010; Cai and Liu, 2015). Although powerful, these methods are hardly adaptable in finer spatial scales applications where the number of images available is lower and the temporal sampling is irregular. However, several local scale applications need high frequency of observations at intra-annual basis. Mapping and monitoring natural and agricultural areas with an enhanced revisit capacity allows monitoring phenology states, agricultural practices and seasonal processes. Recent reviews about conservation monitoring (Nagendra et al., 2013) and Natura 2000 habitat monitoring (Vanden Borre et al., 2011) pointed out remote sensing as a strong, but still underexploited, tool.

In the literature, methods used to process multitemporal optical imagery are commonly grouped under the change detection label. In a pioneer review article, Singh (1989) defined change detection as the process of identifying differences in the state of an object or phenomenon by observing it at different times. The author also categorised the main change detection techniques in ten different groups. A critical review about change detection methods in ecosystem monitoring was provided by Coppin et al. (2004). More recently, Hussain et al. (2013) expanded the change detection categories previously proposed by Singh (1989), including object-based change detection (OBCD) techniques. Regarding this last point, the works of Chen et al. (2012) and Blaschke (2005) provided a deep overview of the available OBCD methods.

Considering SITS of optical imagery, we can highlight twomain limitations in the current literature. Firstly, most of the existing methods focus their efforts on bi-temporal change detection situations, i.e. the study of temporal evolutions taking place between two dates. Usually, these methods include post-classification comparison (Yuan et al., 2005), image differencing (Lu et al., 2005), composite analysis (B. Descle, 2006), linear transformation (Qin et al., 2013) and change vector analysis (Malila, 1980). Secondly, the majority of works explored mainly pixel-based strategies (Petitjean et al., 2012; Inglada et al., 2015) whereas object-based image analysis (OBIA) are still among open challenges in remote sensing analysis (Blaschke et al., 2014; Chen et al., 2012).

Petitjean et al. (2012) constructed vector images from SITS and used classical unsupervised classification (k-means) at pixel level. The originality of the approach consisted in the integration of spatial relationships between pixels. Each pixel was enriched by some 
contextual attributes coming from individual image segmentations performed at each timestamp. In this case, the temporal behavior (based on 15 FORMOSAT-2 images acquired in the same year) was used to assign a unique land cover label (mainly crops) to each pixel. These labels, derived from ground reference data, are static (e.g. corn) and do not describe dynamics (e.g. bare soil $->$ growth of corn $->$ harvest); therefore it is not possible to perform further analysis, or monitoring, related to the intra-annual evolutions. Inglada et al. (2015) evaluated the performance of state-of-the-art supervised classification methods for generating accurate crop type maps on 12 sites spread all over the world. The classification strategy giving the best results combined pixel-based temporal linear interpolation and feature extraction (radiometry derived features only). In this case, SITS were composed of a variable number of SPOT-4 and Landsat-8 images (from 9 to 41 images depending on the site) acquired in the same year. In general, important amounts of ground reference data (from several dozens to a few thousands of hectares) were necessary for training the classifier and achieving accurate results. Also here, the process chain generates a single outcome (i.e. a map) representing static land cover classes. This flat representation, alone, is not able to describe the evolutions and the temporal behaviors behind each class label.

Differently from previous approaches that mainly focus on the classification and/or detection of abrupt changes between consecutive images, this paper aims to describe a new methodology to explore SITS data detecting and describing spatiotemporal entities/phenomena existing in the study area. More in detail, given a time series of remote sensing images and an associated segmentation, our objectives are to: (i) detect the set of spatiotemporal entities/phenomena existing in the study area and (ii) supply a spatiotemporal description for each of them. To this end, we propose an hybrid methodology combining OBIA and data mining techniques. Our proposal firstly identifies a set of spatial entities covering as much as possible the whole study site and, subsequently, for each of those spatial entities, it builds an evolution graph to describe its temporal evolution.

We applied our approach on two study sites involving different types of natural, seminatural and agricultural areas. Since the task we address is completely exploratory and different from most of the previous researches on SITS data (e.g. change detection, classification), to verify and assess the quality of our proposal we performed in-depth qualitative evaluations on the set of evolution graphs we extracted. More in detail, we showed how the evolutions graphs well summarize the temporal profiles of the extracted spatiotemporal phenomena and how they can be employed to synthesize the evolutions and temporal behaviors extracted from a SITS.

The rest of the paper is organized as follows: Section 2 describes all the methodological steps of the proposed approach. Section 3 presents the study case context, namely the time series data, the preprocessing steps and the verification strategies. Experimental results are presented and discussed in Section 4. Conclusions are drawn in Section 5. 


\section{Methodology}

\subsection{Object-based temporal evolutions}

The type of phenomena we want to capture are spatiotemporal evolutions (and their related dynamics) describing how an entity (i.e. a lake, a saltmarsh area, a crop field, etc..) evolves along the time. To this purpose, within a given study site, the first goal of our approach is to automatically detect a set of spatiotemporal entities. Subsequently, a high-level description is constructed for each of those entities employing a graph-based representation. The general framework of our methodology is summarized in Figure 1.

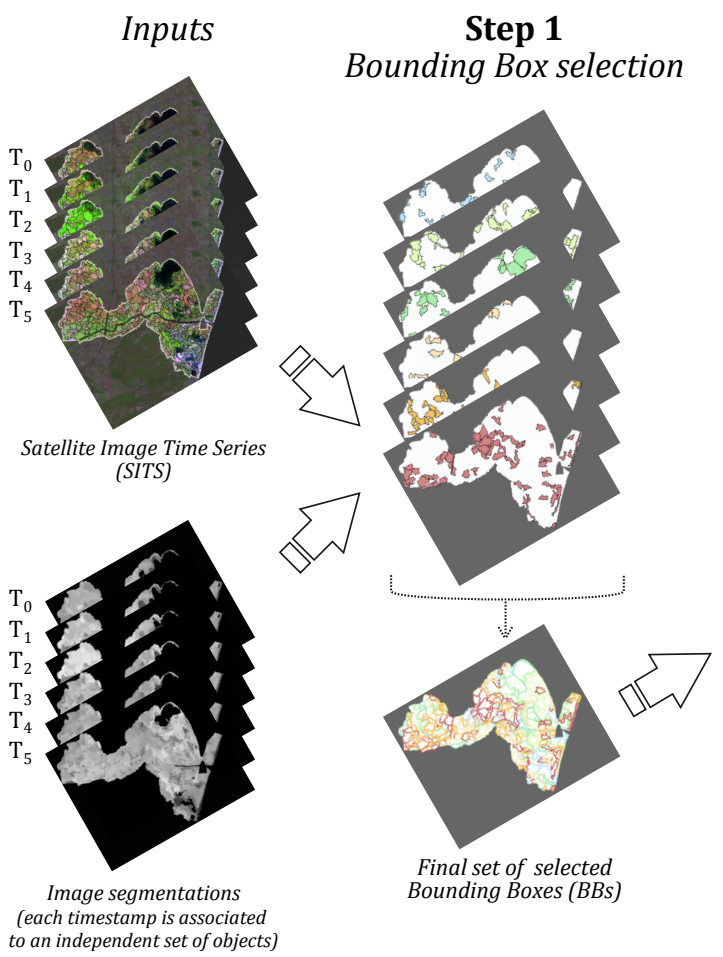

\section{Step 2 Graph construction \\ Step 3 \\ Measuring spatiotemporal evolutions}

Graph level

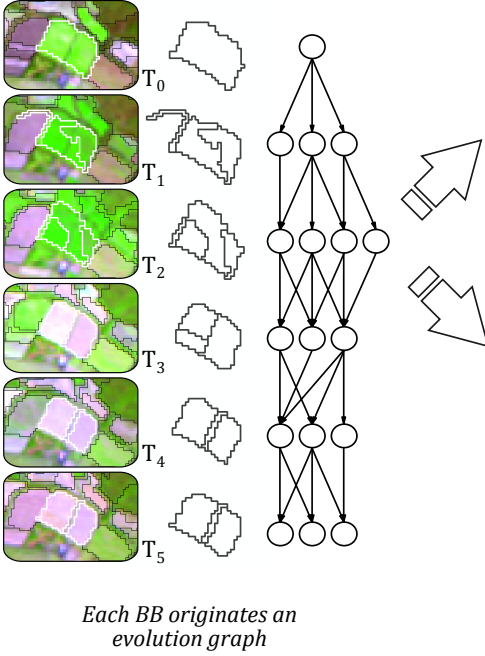

Study-site level

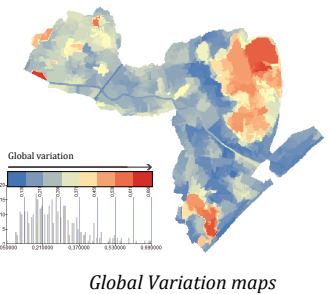

Figure 1: General framework showing the main steps of the methodology.

Given a SITS data and its associated segmentation, firstly we select a set of objects that represent the spatial entities we want to monitor during the time. We call such subset of objects Bounding Boxes $(B B s)$. The set of $B B s$ can contain objects coming from any timestamp. The term spatial entities is used in this paper to designate a part (any portion) of a given study site. Then, for each Bounding Box $(B B)$, we create an evolution graph considering all the objects, in all the timestamps, that are covered by the $B B$ area. Each vertex of a graph corresponds to an object. Two vertices are linked by an edge if they belong to two successive timestamps and the corresponding objects overlap each other. The procedure is applied to each $\mathrm{BB}$ and the result consists in a set of evolution graphs summarizing the different spatiotemporal phenomena existing in the study site. The set of evolution graphs is successively exploited, with the object related information (e.g. spectral, geometrical, textural, etc.) in order to supply analysis at graph and study-site levels. The first level allows 
namely the analysis of the temporal trajectories (or profiles) of a particular spatiotemporal phenomenon while the second level supplies a more general picture summarizing the temporal dynamics detected over the entire study site.

\subsection{Bounding Box selection}

The first step of our process consists in the selection of coherent $B B s$ (i.e. spatial entities) to monitor along the different timestamps. This operation analyzes all the objects provided by the input segmentations (all the timestamps) and selects a subset of different spatial entities covering as much as possible the whole study site. To deal with this task we made some assumptions that are justified from the nature of the SITS data we manage.

The first assumption we made is related to the fact that each selected BB has, during the period considered by the SITS, a maximal extent (or footprint) from a spatial point of view. For instance, if we consider a temporary lake, in the time series we will have a timestamp in which it reaches its maximal spatial extent while for the other timestamps the same area may be segmented in different objects as water will cover a less important area. In our approach we attempt to select maximal footprints as $B B s$. To select the set of $B B s$, we adopted the following strategy: first we select a subset of the objects respecting the assumption on the maximal footprint, we named such set of objects candidateBB. Then, from candidate $B B$ we filtered out a subset of objects that cover as much as possible the study site and that overlay as less as possible between each other from a spatial point of view.

Since all the images span over the same grid of pixels, we can retrieve for each pixel of each timestamp the object it belongs to and therefore select the largest one. The process is repeated over the whole study site and the selected objects are added to candidateBB. This pre-selection explicitly implements the maximal footprints assumption over the whole study site. However, this process may retain objects representing very similar geographical areas. To deal with this redundancy issue we designed an algorithm that, starting from candidate $B B$, selects a set of objects to minimize as much as possible the degree of overlay. More in detail, the algorithm iterates over the set of candidate $B B$ until no more objects can be included in the final set of $B B s$. At the beginning, the set of $B B s$ is initialized to the empty set. A data structure containing the grid pixels covered during the process is initialized with the empty set. We call this structure PAC (Pixel Already Covered). At each iteration, the more promising object is selected from the candidate $B B$ and added to the final set of $B B s$. The more promising object is determined considering the following piecewise function (1):

$$
\text { weight }(O)= \begin{cases}\operatorname{size}(O) & \text { if novelty }(O)=1 \\ \text { novelty }(O) & \text { if } \alpha \leq \text { novelty }(O)<1 \\ 0 & \text { if novelty }(O)<\alpha\end{cases}
$$

where:

- $\operatorname{size}(O)$ is the size of the object, in this case the number of pixels 
- $\operatorname{novelty}(O)$ is the contribution of the object w.r.t. the current partial solution

- $\alpha$ is a threshold parameter defining the minimum value of novelty an object must show to be added to the final set of $B B s$

1.

More in detail, the novelty numerically describes the contribution of each object (belonging to candidate $B B$ ) w.r.t. the partial solution achieved by the procedure. The novelty is defined as follows (2):

$$
\operatorname{novelty}(O)=\frac{|\operatorname{size}(O)-P A C(O)|}{\operatorname{size}(O)}
$$

where:

- $\operatorname{size}(O)=$ the number of pixels of the object

- $P A C(O)=$ the number of pixels already covered by the current partial solution for a given object

In summary, the weight assigned to each candidate $B B$ object is dynamically recomputed during the procedure. This is done because we update the $P A C$ variable whenever an object is added to the final set of $B B s$. According to the weight $(O)$ function, first we will select all the bigger and non-overlapping objects from candidate $B B$ as their novelty value is equal to 1. Then, we will start to select the objects presenting the higher novelty values in order to fill the remaining uncovered areas of the study site. The process stops when all the remaining candidate $B B$ objects present novelty values lower than the parameter $\alpha$ or all the grid pixels of the study site are covered. The value of $\alpha$ is inversely proportional to the number of $B B s$ in the final set. High values of $\alpha$ will lead the selection of a small set of $B B s$, while small values of $\alpha$ will allow the procedure to extract a bigger set of $B B s$. Another point we can stress on is that the $B B s$ extracted with a big value of $\alpha$ (e.g. 0.5) will be a subset of the $B B s$ extracted with a small value of $\alpha$ (e.g. 0.3). This is due to the fact that the proposed procedure is deterministic and has a monotonic behavior. Decreasing the value of $\alpha$ will relax the spatial overlay constraint going further in the selection process.

\subsection{Graph construction}

The final set of $B B s$ defines the spatial entities (and their related phenomena)we will monitor throughout the SITS. Logically, each $B B$ has a unique spatial extent (footprint) which is used to select and link the objects from one timestamp to the next one. Given a $B B$, we project its footprint over each timestamp of the time series and we select the objects overlapping with $B B$. In order to avoid the selection of non-representative objects (or parasite objects) w.r.t. the area we monitor, we established two parameters that can be translated to the following restrictive conditions: (a) at least $\tau_{1}$ of the object should be inside of the $B B$ footprint, (b) the object should represent at least $\tau_{2}$ of the $B B$ footprint where both $\tau_{1}$ and $\tau_{2}$ are two percentages. The first parameter $\left(\tau_{1}\right)$ is the most important 
and control the selection of objects that should present most of their spatial extent outside the $B B$ footprint. The second parameter $\left(\tau_{2}\right)$ is used to keep all the objects filling more than a certain percentage of the $B B$ footprint, irrespective of any other statement.

After this selection, each $B B$ will be associated to a set of objects which can be organized and stored as an evolution graph. The graph is built linking the objects of timestamp $i$ with the objects of timestamp $i+1$. Each object corresponds to a vertex of a graph and the weight of the link (edge) represents the degree of overlap between two objects. In this way we obtain graphs that have as many layers as the number of images in the time series. Another intrinsic characteristic of an evolution graph is that, for a certain layer, it will contain only one object (corresponding to the $B B$ ). Logically, objects belonging to the same timestamp are not connected; this is also true for objects not belonging to two successive timestamps. In other words, the graphs created by our procedure are oriented graphs, more precisely temporal oriented graphs. An oriented graph is the same thing as a loopless simple directed graphs (West, 2001), also called Directed Acyclic Graphs (DAGs) (Maurer, 2003).

\subsection{Computing graph coverages}

Each evolution graph is associated to an unique $B B$ and can be represented by several spatial coverages (see Figure 2). The simplest way is to use the spatial extent of the former $B B$ to represent the graph (e.g. in a map). We named this representation the Bounding Box Graph Coverage (BBCov). In order to get a Whole Graph Coverage (WholeCov) we calculated the total spatial extent of all the objects contained in the graph at all the timestamps. The WholeCov can be decomposed in two components, the Ephemeral Graph Coverage (EphemCov) which groups the area(s) covered only once during the time series and the Core Graph Coverage (CoreCov) which indicates the area(s) covered at least twice during the time series. Such surfaces (EphemCov and CoreCov) can be expressed as percentages of the WholeCov. High percentages of EphemCov indicate unstable boundaries of the graph objects and can be related to transitory evolutions in the study area. However, sometimes this behavior can be produced by unsuitable segmentation results, e.g. under segmentation. In such a case the input segmentation can influence the extraction of interesting evolution graphs. This means that the EphemCov value can be employed as an indicator to estimate the quality of the time series segmentation and suggest, if necessary, to provide a better input segmentation that will impact positively the graph coverage results. How to optimize and produce coherent individual segmentations from a SITS is out of the scope of this work since, these two elements (SITS and segmentations) are the inputs of the proposed methodology. CoreCov usually encompasses the whole surface of the BBCov as well as a buffer area around it. A big discrepancy between CoreCov and BBCov usually indicates that the $B B$ does not provide a good spatial representation of the whole graph.

In the example showed in Figure 2, the $B B$ used to create the evolution graph comes from the first timestamp $\left(T_{0}\right)$ and its coverage $(B B C o v)$ is highlighted in orange color. It represents two agricultural parcels covered by the same type of crop. In the following timestamps, the number of objects ranged from 3 to 4 and the evolution graph totaled 17 objects. The union of all these objects corresponds to the WholeCov which is showed in black. We can notice an elongation in the upper left part of the WholeCov polygon if 


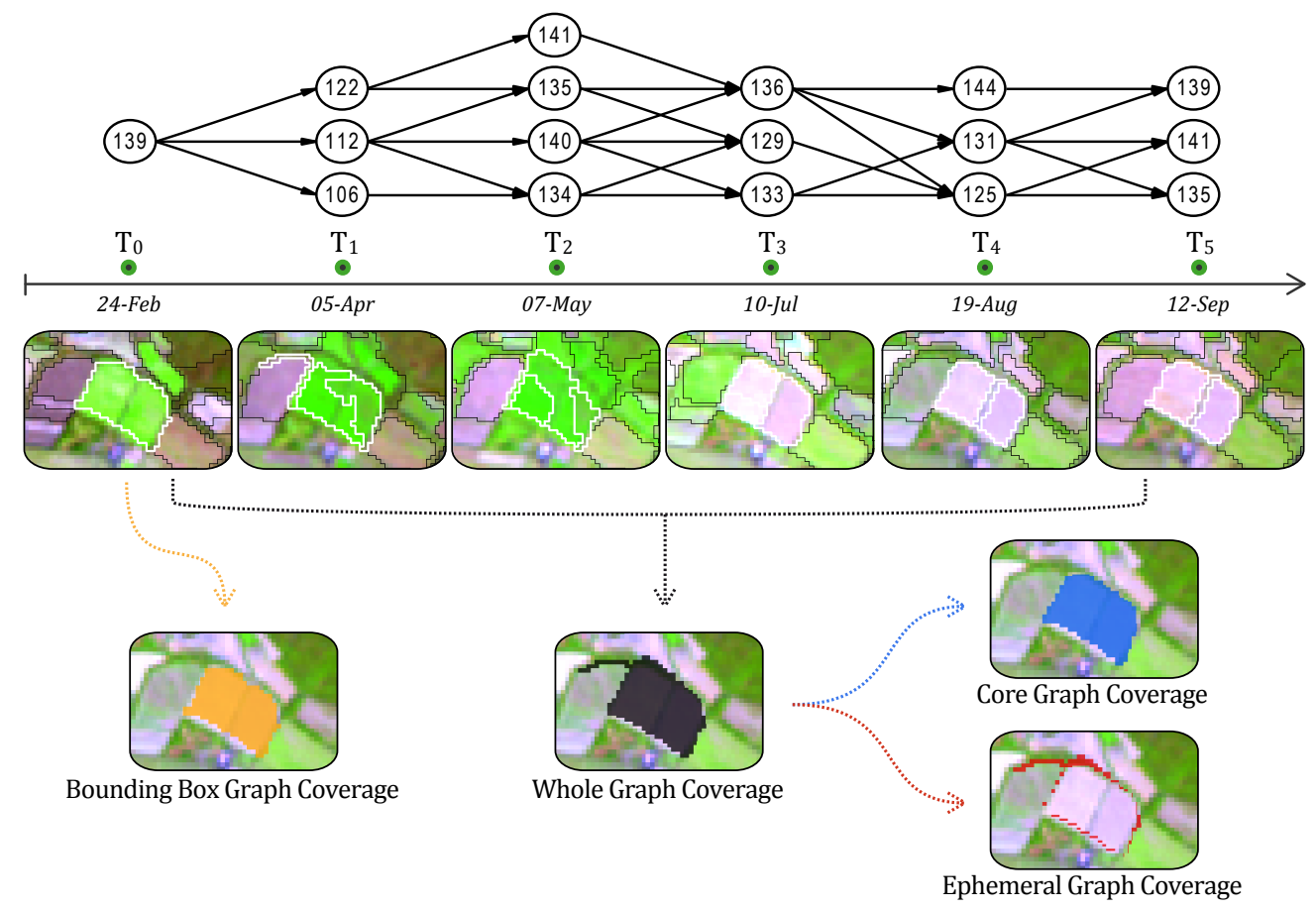

Figure 2: Example of an evolution graph extracted from a crop area. Graph nodes and edges are showed in the upper part, while the object boundaries (at each timestamp) are displayed below the timeline. The four types of spatial coverages computed for this evolution graph can be visualized in the bottom part of the figure.

compared to $B B C$ ov. The elongation does not correspond to the agricultural parcels targeted by the $B B$ but to a parasite object coming from $T_{1}$. This undesirable inclusion is clearly visible in the EphemCov (red color) which highlights also other small border sections around the agricultural parcels. In this example, the CoreCov (blue color) is very similar to the $B B C$ ov as the borders of the targeted parcels remained substantially stables during the time series.

\subsection{Measuring spatiotemporal evolutions at Graph and Study-site levels}

In order to analyze and understand the information behind the evolution graphs, we defined two levels of analysis: (a) the graph level and (b) the study-site level. In the former, the focus is mainly on how the objects of the graph are linked (graph structure) and how their attributes (content) evolve in time. In the latter, the focus is related to the whole study site and especially on how the most stable and the most dynamic spatial entities are distributed.

Considering the graph level analysis, given a graph $G$, we indicate with $G_{i}$ the set of objects covered by $G$ at the timestamp $i$ and with $w_{j, k}$ the weight of the link between object $o_{j}$ and object $o_{k}$. We compute the Variation ( Var) between two consecutive timestamps with the following formula (3): 


$$
\operatorname{Var}\left(G_{i}, G_{i+1}\right)=\sum_{o_{j} \in G_{i}} \frac{\operatorname{size}\left(o_{j}\right)}{\operatorname{size}\left(G_{i}\right)} \cdot \frac{\sum_{o_{k} \in G_{i+1}} w_{j, k} \cdot \operatorname{dist}\left(o_{j}, o_{k}\right)}{\sum_{k} w_{j, k}}
$$

The first part of the $V$ ar formula is proportional to the importance of the object $o_{j}$ over the set of objects at timestamp $i$. Therefore, size $\left(o_{j}\right)$ corresponds to the number of pixels of $o_{j}$ while size $\left(G_{i}\right)$ represents the total number of pixels covered by the graph $G$ at timestamp $i$. The second part of the formula evaluates the evolution between an object at timestamp $i$ w.r.t. the objects at timestamp $i+1$ linked to it. In particular, the variation between two timestamps is measured by a weighted sum of the euclidean distances between the attributes of the object $o_{j}$ and $o_{k}$. The weight $w_{j, k}$ quantifies the strength of the interaction between $o_{j}$ and $o_{k}$ in terms of spatial overlay.

The Global Variation (GlobaVar) for a graph is obtained cumulating the contribution of each pair of consecutive timestamps as follows (4):

$$
\operatorname{GlobalVar}(G)=\sum_{i=1}^{n-1} \operatorname{Var}\left(G_{i}, G_{i+1}\right)
$$

The GlobalVar associated to an evolution graph estimates how much the area represented by this graph evolves during the period covered by the time series. Potentially, this score can vary between 0 and $+\infty$. A low value of GlobalVar implies stable temporal behavior while a high value indicates important temporal evolution throughout the time series.

Another option to perform graph-level analysis is by means of Temporal Profiles where the temporal variation of any object attribute can be plotted for all the nodes of a given graph. Temporal Profiles allow a more fine analysis of the graphs, facilitating the visualization and interpretation of temporal behaviors related to the graphs' underling spatiotemporal phenomena. More in detail, given a graph $G$ and an attribute we want to monitor (e.g. the NDVI), we can build a plot where the X-axis (resp. the Y-axis) represents the time (resp. the attribute to study, i.e. NDVI). Such plot will contain the objects of the graph temporally arranged from the first to the last timestamp. Such a representation combines the graph structure and the content (i.e. the attribute chosen to perform the analysis), allowing to follow the evolution of these elements conjointly all over the SITS. Examples of Temporal Profiles are reported in the experimental results (see Figure 8).

Considering the study-site level analysis, the GlobalVar scores (computed for each evolution graph) can be used to produce a GlobalVar map. In this kind of representation, any of the computed graph coverages (e.g. CoreCov) can be used to construct the map. According to the selected coverage, the polygons representing the graphs will be colored following a gradient proportional to their GlobalVar scores. The GlobalVar map summarizes the distribution of the different phenomena detected within the study site and provides information related to the intensity of the evolutions during the time. This kind of map, computed automatically and considering the whole SITS, is an useful tool for exploratory researches over areas where the spatiotemporal dynamics are unknown (or few studied). GlobalVar maps can also provide valuable information for planning field-campaigns and prioritizing the visits over such unknown or few studied areas. In the case of similar temporal sampling, 
GlobalVar maps may be used to compared the spatiotemporal dynamics of two (or more) different study sites.

While the Temporal Profiles are more suitable for analyzing one particular object attribute at time, GlobalVar scores (and maps) can be also obtained considering all the attributes or a subset of them (e.g. only a few spectral indices or a given combination of spectral bands).

It is important to highlight that the GlobalVar score is more suitable for short-term landscape analysis (e.g. intra-annual scale) and less appropriate for long-term landscape evolution monitoring (e.g. multi-annual scale) since different temporal trajectories can collapse to the same score value. Conversely, the information supplied by Temporal Profiles can be adopted to study both short-term or long-term landscape evolutions since it preserves the full temporal trajectories associated to an evolution graph.

\subsection{Parameter Setting}

As previously noticed, our methodology needs the setting of three different parameters: $\alpha, \tau_{1}$ and $\tau_{2}$. The first parameter limits the overlay among the selected $B B s$ while the remaining two parameters avoid the selection of non-representative objects in the construction of the evolution graphs.

With the aim to facilitate the choice of these parameter values, we propose to consider the coverage and the redundancy of the extracted evolution graphs. The coverage of the evolution graphs is the union of the WholeCov of each of the graphs in the solution. This measure quantifies how much of the study site is covered by the selected graphs. Concerning the redundancy in the set of extracted graphs, we evaluate this quantity as the portion of the study site that is covered, at least, by two different graphs. This quantity measures how much redundancy exists in the obtained solution.

In order to determine the three initial parameters (and the corresponding set of evolution graphs), we firstly generate different solutions varying the $\alpha, \tau_{1}$ and $\tau_{2}$ parameters and then, we fix a threshold $(\sigma)$ that defines the minimum accepted coverage. The $\sigma$ threshold is expressed as a percentage of the whole study area. Once the threshold $\sigma$ is fixed, we obtain a set of solutions that meets this constraint. Among such set of solutions, we choose the one with the minimum redundancy value. We remind that this analysis can be performed in a completely unsupervised way, independently from a possible ground truth data associated to the SITS.

\section{Case study}

\subsection{Data and Study sites}

\subsubsection{Time series data}

We used Landsat-5 TM and Landsat-7 ETM+ level-2A products available through the THEIA Data Centre (France). Such images were already ortho-rectified and corrected from atmospheric, environmental and slope effects as described by Hagolle et al. (2010). Each Landsat product was composed by six spectral bands (approximate center in $\mathrm{nm}$ ): blue (485), green (565), red (665), NIR (820), SWIR-1 (1650) and SWIR-2 (2190). With a pixel size of 
$30 \mathrm{~m}$, the raster data is expressed in surface reflectance. We selected six Landsat cloud-free images covering two study sites (described latter) between February and September 2009 (see Table 1).

\begin{tabular}{cc}
\hline Timestamp & Acquisition date \\
\hline$T_{0}$ & 24 Feb. 2009 \\
$T_{1}$ & 05 April 2009 \\
$T_{2}$ & 07 May 2009 \\
$T_{3}$ & 10 July 2009 \\
$T_{4}$ & 19 Aug. 2009 \\
$T_{5}$ & 12 Sept. 2009 \\
\hline
\end{tabular}

Table 1: Acquisition date of the selected Landsat images over the South of France.

The selected time series spreads from the end of the winter up to the end of the summer. Such temporal range encompasses the entire growing season for natural vegetation as well as the main agricultural cycles over the study sites.

\subsubsection{Study sites description}

Two sites were selected in the south of France, close to the Mediterranean Sea. Figure 3 presents the spatial boundaries of the two sites: (A) Libron Valley and (B) Lower Aude Valley Natura 2000 site. Both sites are located inside the extent of the Landsat scenes composing our time series. Figure 4 shows the study areas at each timestamp.

Located less than $10 \mathrm{~km}$ northeast from the city of Béziers (France), the Libron Valley site is mainly composed by agricultural parcels and natural areas. The site has about 1655 ha and is crossed by the small coastal river named Libron. Agricultural parcels are concentrated principally along the Libron waterway. Cereal crops dominate its upstream section (northwest of the site) while the downstream section is mainly occupied by vineyards (southeast of the site). The natural areas are essentially composed by patches of forest (mainly coniferous) and scrubland. Most of these patches are in the north of the Libron River, some of them encircle a golf field situated in the northern part of the site. In a general way, the limits between agricultural and natural areas over this site can be easily recognized in the Landsat images. Such a task is possible because agricultural parcels and forest patches are usually bigger than 6-8 ha (i.e. $200 \mathrm{~m} \mathrm{x} 400 \mathrm{~m}$ or wider for most of the crop fields).

The Lower Aude Valley is a Natura 2000 site located in the terminal section of the Aude River. Before reaching the Mediterranean Sea, the Aude River crosses a flat wetland area of about 4842 ha. From a biodiversity point of view, $56.3 \%$ of the site is composed of natural habitat types of Community interest (NHCI). In total, 19 NHCI are part of the site, including 5 priority habitat types. The most widespread habitats are: Mediterranean saltmarshes and Saline coastal lagoons. The remaining area $(43.7 \%)$ is principally occupied by vineyards, cereal crops and temporary or permanent meadows. In opposition to the Libron site, the agricultural parcels are often small within this site (usually around 1-2 ha) and therefore more difficult to identify using Landsat images. Another particularity, the site is exposed to 


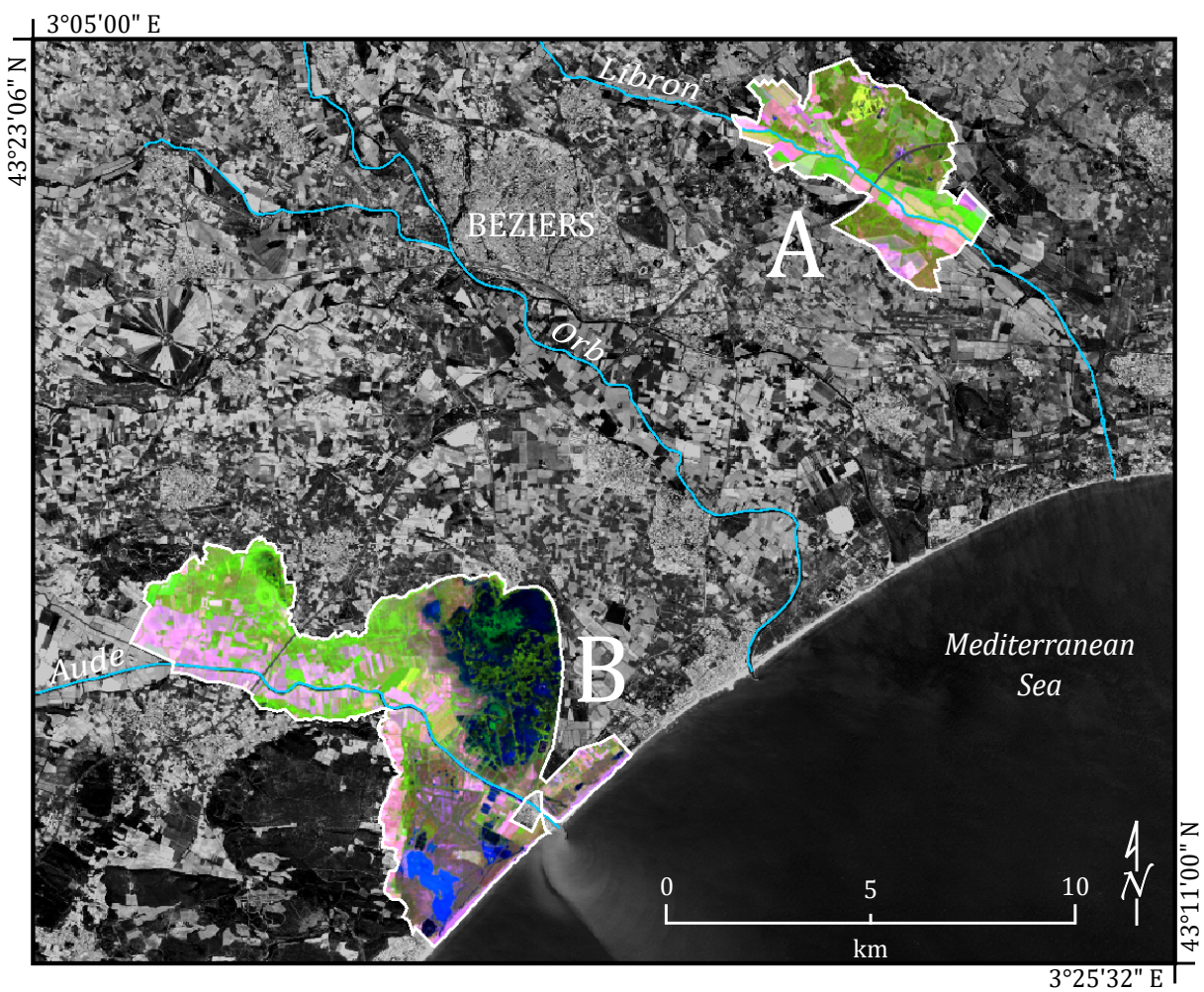

Figure 3: Location and boundaries of the selected study sites (A Libron Valley ; B Lower Aude Valley Natura 2000 site).

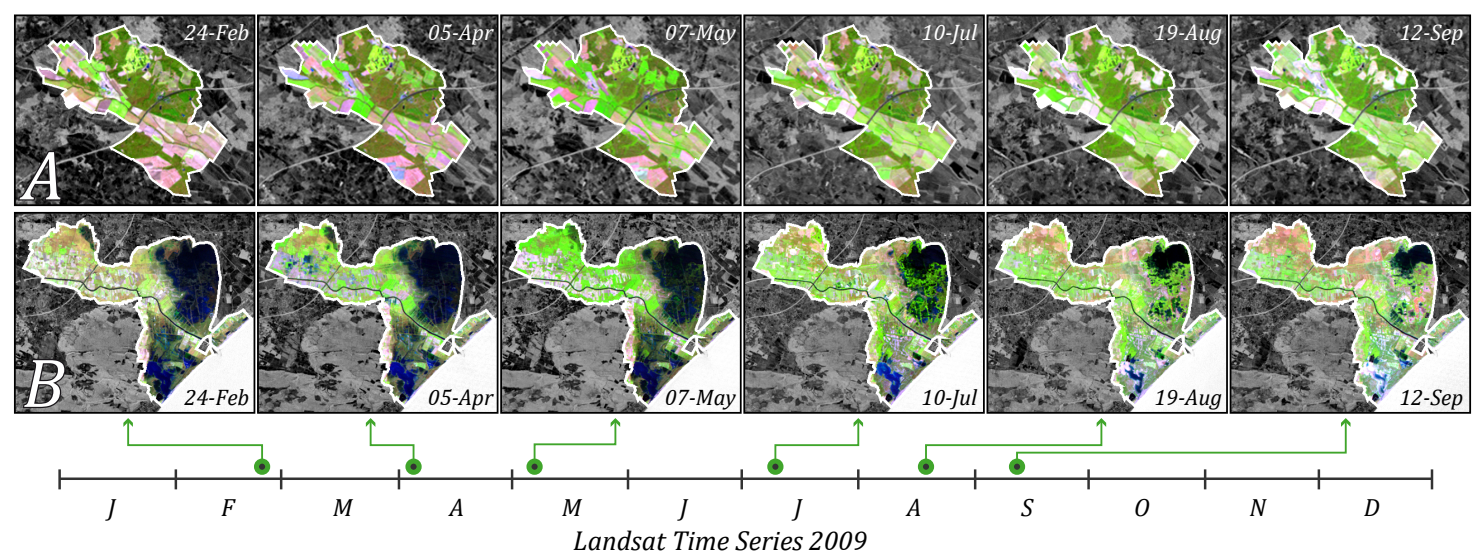

Figure 4: Time series for the selected study sites (A Libron Valley ; B Lower Aude Valley Natura 2000 site) during 2009.

flooding events (mostly during winter) as well as to drought episodes (maximum intensity occurring in the end of the summer). The flooding areas are situated predominantly around the two coastal lagoons: Vendres in the north part of the site and Pisse-Vaches in the south. The Mediterranean Sea has also an influence over the salinity across the site (soils and water bodies), with a general gradient increasing from northwest to southeast. 


\subsection{Preprocessing and segmentation}

\subsubsection{Spatial subset and fine geometrical registration}

Although level 2-A products were already ortho-rectified, we observed some spatial imprecision when overlapping all the time series images. For this reason, additional fine spatial positioning corrections were necessary in order to keep the spatial shift between any timestamp less than a pixel. Afterwards, two spatial subsets (one for each study area) were performed over each Landsat image.

\subsubsection{Spectral indices}

Spectral indices are commonly used in remote sensing as they can be helpful for detecting and characterizing some specific features, like vegetation, soil, water, etc. In this work we calculated three spectral indices compatible with Landsat data using the formula provided by the literature: a) Normalized Difference Vegetation Index NDVI (Rouse Jr et al., 1974); b) Normalized Difference Water Index NDWI (Gao, 1996); c) Visible and Shortwave Infrared Drought Index VSDI (Zhang et al., 2013). NDVI is sensitive to the amount of photosynthetically active vegetation present in the plant canopy (Tucker, 1979) and has been extensively used in remote sensing applications since the 1970s. NDWI is sensitive to changes in liquid water content of vegetation canopies (Gao, 1996) and has been used to estimate vegetation water content (Jackson et al., 2004). VSDI is sensitive to changes in soil and vegetation moisture and was conceived to monitor drought over different types of land cover during plant-growing season (Zhang et al., 2013).

\subsubsection{Time series image segmentation}

Image segmentation is a fundamental step in OBIA and it consists in merging pixels into object clusters (Baatz et al., 2008). Objects (or segments) are regions generated by one or more criteria of homogeneity in one or more dimensions of a feature space (Blaschke, 2010). The principal aim of segmentation is to create a new representation of the image, more meaningful and easier to analyze. This approach is similar to human visual interpretation of digital images, which works at multiple scales and uses color, shape, size, texture, pattern and context information (Lillesand et al., 2008). Image segmentation results in a set of objects that collectively cover the entire image without any overlapping. With respect to the homogeneity criteria, adjacent objects are expected to be significantly different between them.

In this work, image segmentation was performed with the Multiresolution Segmentation Algorithm (MSA) ${ }^{1}$. We choose the MSA algorithm instead of recent approaches based on superpixel Achanta et al. (2012) since the objective of our strategy is to capture phenomena that can lie at different scales. Adopting a superpixel segmentation method, like SLIC (Achanta et al., 2012), will produce segments at equal scale and this will be in contrast with the main assumption of our work (maximal spatial extent detection). Conversely, the MSA scale parameter is intrinsically related to the homogeneity criterion which takes

\footnotetext{
${ }^{1} \mathrm{MSA}$ algorithm: as implemented in eCognition Developer software, version 8.8.1
} 
into account both shape and radiometry of objects in a combined manner. For this reason, over two areas of the same size, MSA may provide multiple small objects if the target is heterogeneous or, a single larger object if the target is more uniform.

Only the pixels within the boundaries of the study sites were used during the segmentations. Nine raster layers were simultaneously used for image segmentation. Six of them correspond to the Landsat spectral bands and the other three to the spectral indices. In order to obtain objects representing the natural and agricultural boundaries over the study sites, we conceived a segmentation rule-set composed of 3 main steps as showed in Figure 5. For simplification purposes only the Lower Aude Valley site is presented in this figure as well as in the subsequent explanations. However, the same rule-set was applied over the Libron Valley site.

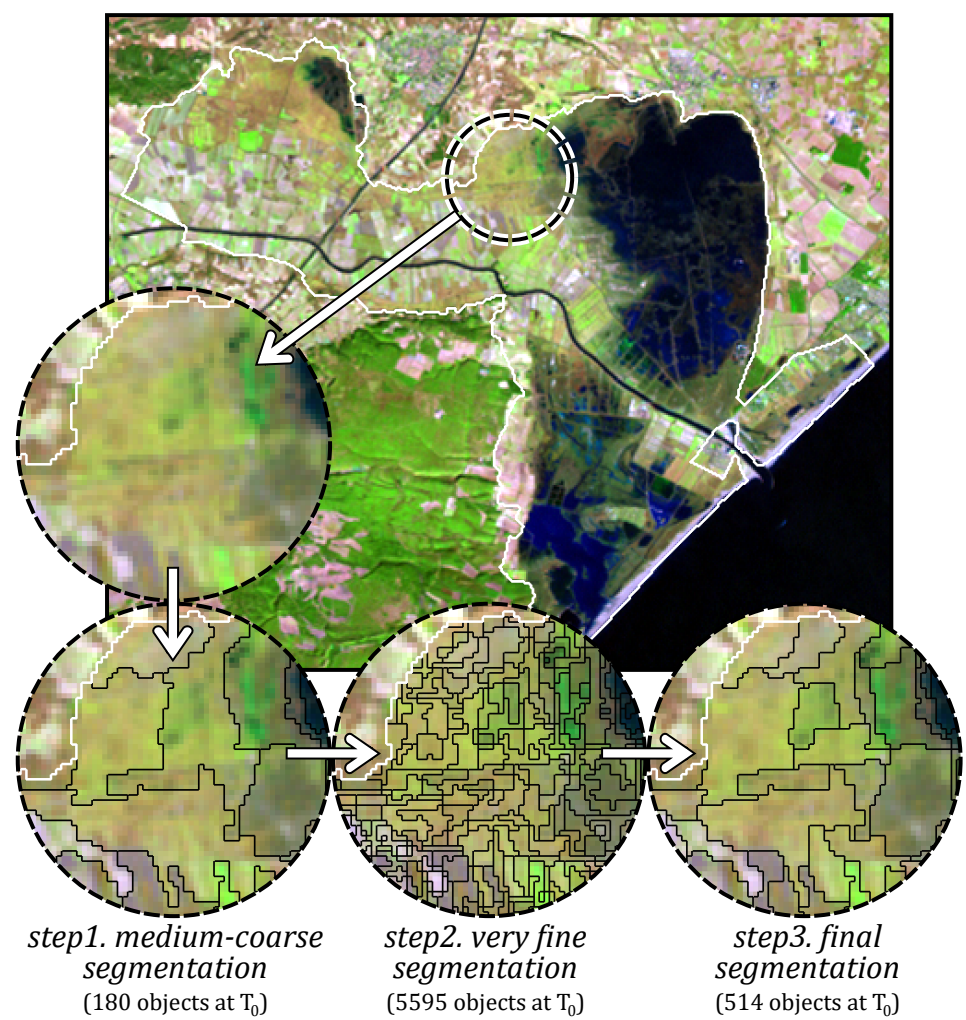

Figure 5: Segmentation rule-set outputs (at $T_{0}$ ) for the Lower Aude Valley Natura 2000 site.

The first step delimits the general zones trough a medium-coarse segmentation. MSA was configured here to combine both color and shape components but using predominantly color (0.8). About 170-200 objects are obtained per timestamp over the Lower Aude Valley site. In the second step, a very fine segmentation is performed inside the object boundaries created at step 1. Focused exclusively on the color component, it creates about 6000 objects per timestamp. In step 3, medium-fine segmentation is performed taking into account the results of the previous steps. With balanced weights for color and shape components, step 3 segmentation creates about 500-600 objects per timestamp. The segmentation rule-set was 
executed for each timestamp separately. In other words, the set of objects obtained at $T_{0}$ does not impact the segmentation process at $T_{1}$ and so on. The segmentations were also separately performed over each study site. For both sites and timestamps, only the objects obtained at the last level of segmentation (step 3) were exported and used as an input for the subsequent processing steps.

\subsection{Verification strategies}

To assess the quality and the accuracy of the results, we developed two verification strategies. The first was based on the interpretation of the ancillary imagery plus two thematic layers and was applied over the Lower Aude Valley site. The second strategy was mainly based on the official farmer declarations (one of the available thematic layer) and applied over the Libron Valley site.

Considering the ancillary imagery, two types of image were employed: (a) normal color and color infrared aerial orthophotos ( $0.5 \mathrm{~m}$ spatial resolution) acquired during May 2009 and (b) one RapidEye satellite image (6.5 m spatial resolution) acquired in 24 June 2009 and only available for the Lower Aude Valley site.

Regarding the thematic layers, the first concerns both study areas and is related to agricultural practices. It corresponds to the official farmer declarations indicating the main cultures exploited during 2009. The second thematic layer is proper to the Lower Aude Valley site. It corresponds to a detailed classification (scale 1:25000) of the natural habitats over the site. The classification was realized by botanists and ecologists of the Conservatory for the Natural Spaces of the Languedoc-Roussillon Region (CEN-LR).

\subsubsection{Ancillary imagery based verification}

First, the aerial photographs were used to map the whole Lower Aude Valley Natura 2000 site. This task was carried out through a manual land cover digitalization process at the 1:10,000 scale. Each individual map unit (polygons in our case) has been labeled according to hierarchically structured land cover classes. This hierarchy contains three levels of complexity and has, in the more detailed level (3), nineteen land cover classes. Eleven of them are associated to artificial, cultivated and managed areas, while the other eight classes are related to natural and semi-natural areas (see Table 2 for all the class names). Considering the acquisition date of the aerial photographs, the obtained land cover map represents the situation of the site in May 2009.

Then, the obtained land cover map was superimposed on the RapidEye satellite image. All the initial polygons received a new land cover label (using the same hierarchical scheme) according to the situation observed on the RapidEye image (24th June 2009). When necessary, new boundaries were digitalized and some polygons of the former map were consequently divided into two or more smaller polygons. Thus, a second land cover map was produced representing the situation of site in late June 2009. In order to estimate the evolutions between the two land cover maps we computed an exhaustive set of from-to evolution classes. Then, we analyzed each from-to evolution class (about 50) and assigned a particular level (or intensity) of change: low, medium, high or very high. Finally, these 


\begin{tabular}{|c|c|c|}
\hline Level 1 & Level 2 & Level 3 \\
\hline \multirow{11}{*}{$\begin{array}{l}\text { Artificial, cultivated } \\
\text { and managed Areas }\end{array}$} & \multirow{2}{*}{$\begin{array}{l}\text { Artificial surfaces } \\
\text { and associated areas }\end{array}$} & Highway and major road sections \\
\hline & & Other built-up and associated areas \\
\hline & Artificial waterbodies & Artificial lakes and ponds \\
\hline & \multirow[t]{8}{*}{ Cultivated and managed areas } & $\begin{array}{l}\text { Crops - dense cover and high } \\
\text { greenness values }\end{array}$ \\
\hline & & $\begin{array}{l}\text { Crops - moderate/sparse cover and } \\
\text { high greenness values }\end{array}$ \\
\hline & & Crops - low greenness values \\
\hline & & Crops - harvested parcels \\
\hline & & $\begin{array}{l}\text { Crops - floating row covers } \\
\text { and bare soils (very high reflectance) }\end{array}$ \\
\hline & & Vineyards - sparse cover \\
\hline & & Vineyards - dense/moderate cover \\
\hline & & Orchards \\
\hline \multirow[t]{8}{*}{ Natural and semi-natural areas } & \multirow[t]{4}{*}{$\begin{array}{l}\text { Natural and semi-natural } \\
\text { vegetation areas }\end{array}$} & $\begin{array}{l}\text { Dense/moderate cover and } \\
\text { high greenness values }\end{array}$ \\
\hline & & $\begin{array}{l}\text { Dense/moderate cover and } \\
\text { moderate greenness values }\end{array}$ \\
\hline & & $\begin{array}{l}\text { Dense/moderate cover and } \\
\text { low greenness values }\end{array}$ \\
\hline & & Sparse cover \\
\hline & Bare areas & Dry flats \\
\hline & \multirow{3}{*}{ Natural areas covered by water } & Unvegetated dunes and beaches \\
\hline & & Shallow waters \\
\hline & & Deep waters \\
\hline
\end{tabular}

Table 2: Hierarchically structured land cover classes used for mapping the Lower Aude Valley site. This scheme was used to create two maps, one derived from the aerial photographs and the other from a RapidEye image.

intensities of change (derived from the ancillary imagery) were compared to the GlobalVar scores, obtained from the evolution graphs (described in Section 2.5).

\subsubsection{Thematic layer based verification (official farmer declarations)}

The second verification procedure consisted in drawing up a parallel between the Global Variation results and the principal groups of culture declared annually by the farmers. In France, the reference parcel representation is the Farmers block/ilot in regard to the European regulation (Comm. Reg. N 796/2004). This kind of parcel representation corresponds to an association of one or more agricultural parcels into blocks. Each block is the property of a single farmer and may contain one or several crop groups (Sagris and Devos, 2008). In practice, the official farmer declarations (the public version of the data) consists in a set of georeferenced polygons (one for each block) were a code indicates the principal groups of culture exploited during the year. Within the Libron Valley site, 11 groups of culture have been declared in 2009 which corresponds to 59 polygons. Nevertheless, this thematic layer contains some erroneous declarations, imprecise polygon boundaries and some gaps (i.e. when an agricultural parcel has not been declared). In order to attain a more precise comparison, we verified each polygon and selected only those without visible errors. Also, we eliminated all the polygons smaller than 4 ha to preserve an order of magnitute comparable with the graph objects. The obtained subset contains 32 polygons belonging to the following groups of culture: cereals (excepted wheat), flower-fruit vegetables, orchard, seeds, sunflower and vineyard. As these cultures are associated to dissimilar agricultural practices 


\begin{tabular}{l|ccc|ccc}
\hline & \multicolumn{3}{|c|}{ Lower Aude } & Valley & \multicolumn{3}{c}{ Libron Valley } \\
& Min & Mean & Max & Min & Mean & Max \\
\hline Number of nodes & 7 & 15.2 & 38 & 6 & 13.0 & 26 \\
Number of edges & 7 & 24.5 & 77 & 5 & 18.9 & 53 \\
Number of paths & 2 & 79.7 & 1050 & 1 & 46.7 & 480 \\
BBCov (ha) & 1.6 & 16.2 & 125.0 & 3.2 & 13.8 & 67.1 \\
WholeCov (ha) & 5.3 & 46.2 & 175.7 & 6.1 & 34.5 & 107.9 \\
CoreCov (ha) & 2.1 & 29.9 & 142.5 & 3.4 & 22.6 & 94.6 \\
CoreCov (\%) & 13.9 & 65.1 & 90.9 & 14.4 & 66.3 & 93.3 \\
EphemCov (ha) & 0.8 & 16.4 & 134.0 & 1.2 & 11.8 & 65.1 \\
EphemCov (\%) & 9.1 & 34.9 & 86.1 & 6.7 & 33.7 & 85.6 \\
\hline
\end{tabular}

Table 3: Global graph statistics obtained for each study site

and temporal dynamics all along the year, it is expected some noticeable differences among the graphs representing these areas (especially w.r.t. the GlobalVar results).

\section{Experimental Results and Discussion}

\subsection{Overall results and statistics}

To generate the evolution graphs on the two study sites we used the procedure introduced in Section 2.6. We fixed the $\sigma$ threshold (the minimum accepted coverage) equals to $95 \%$ and generated the set of different solutions varying the three parameters $\left(\alpha, \tau_{1}\right.$ and $\left.\tau_{2}\right)$ in the range $[0.1,1]$ with a step-size of 0.05 . The procedure selected the following values for $\alpha$, $\tau_{1}$ and $\tau_{2}: 0.3,0.25$ and 0.20 respectively. The values are the same for both sites.

We obtained a total of 340 graphs for the Lower Aude Valley site and a total of 142 graphs for the Libron Valley site. The total number of objects per graph ranges from 6 (a single object per timestamp) to 38 (about 6.3 objects per timestamp). The mean value, considering both study sites, was 14.6 (about 2.4 objects per timestamp). Also considering the two study sites, the mean number of edges per graph was 22.9 while the mean number of paths per graph was 69.9. Taking into account all the 482 graphs, the whole spatial coverages ( WholeCov) ranges from 5.3 ha to 175.7 ha with a mean value of 42.7 ha. Although some graphs present very high coverages (>100 ha), most of the values (about 97\%) range between 10 and 90 ha. In other words, the areas monitored by our graphs correspond mostly to patches ranging from 100 to 1000 Landsat pixels. As another global result, the core graph coverages (CoreCov) correspond, in average, to $65.5 \%$ of the WholeCov areas. As expected, Ephem Cov is usually smaller than CoreCov and this is true for $87.5 \%$ of the graphs. Even if all the processing steps were identical for the two study sites, we noticed some differences in the graph derived statistics. Table 3 shows the main statistical results obtained separately for each study site.

We can observe that the Lower Aude Valley graphs have a bigger number of nodes if compared to those of the Libron site. In general, they tend to present a more complex structure with a higher number of paths per graph. Another noticeable difference is related to the size of the objects and the derived graph coverages. All the greatest objects (>70 ha) 


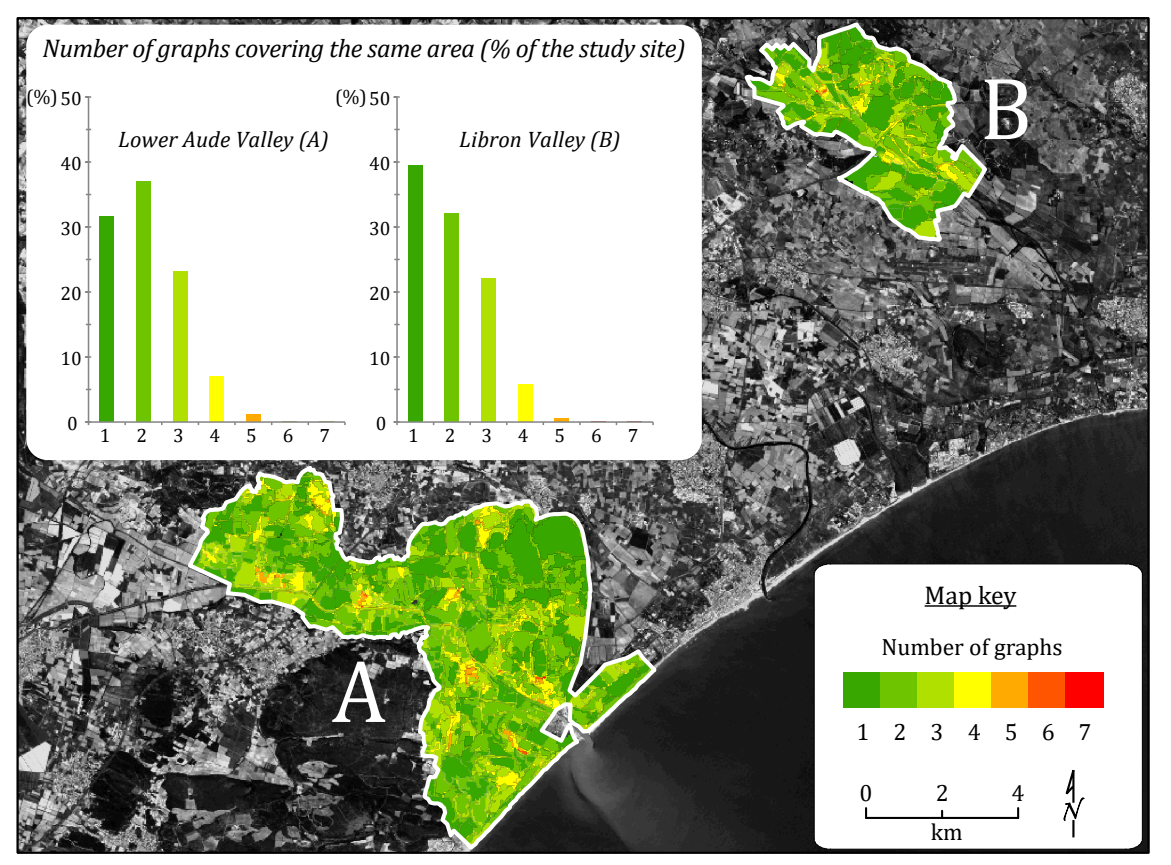

Figure 6: Degree of spatial overlapping among graphs for both study sites. The histogram indicates the relative areas (\% of each study site) considering number of graphs covering the same area.

comes from the Lower Aude Valley as well as most of the widest graphs (>100 ha w.r.t. the Whole Cov). This can be explained by the exclusive presence of water bodies and temporally flooded areas in the Lower Aude Valley site. The spectral homogeneity of these particular areas contributes to generate large objects during the image segmentation step.

As the graph coverages may partially overlap, it becomes interesting to detect the spatial distribution of the less and most overlapping areas. Figure 6 shows such spatial distribution, in terms of number of graphs representing the same area, over the two study sites. The spatial overlapping is related to the value of the parameter $\alpha$ (novelty threshold) used during the $B B$ selection strategy presented in Section 2.2. This threshold allows a certain level of overlay among the $B B s$, it is expected that all the other derived graph coverages will present some degree of overlap as well. As a consequence, the higher is the $\alpha$ parameter, the lower will be the number of selected $B B s$, the lower will be the degree of spatial overlapping among the generated graphs. On both study sites we have observed that, when $\alpha$ is lower than 0.2 the degree of overlay becomes particularly high (more than $75 \%$ of the study site is covered by two or more graphs) while $\alpha$ values larger than 0.4 lead to important gaps (areas not covered by any graph).

However, the spatial overlapping depends also on the inner characteristics of each study site, in particular on how the spatial boundaries of their objects evolve during the time series. In the case of our study sites, the Libron Valley presented a lower degree of spatial overlapping w.r.t. the Lower Aude Valley. This can be explained by two main factors: (a) the spatial arrangement of the sites, e.g. in the Libron site the limits between agricultural and natural areas are easier to recognize (bigger and more homogeneous patches) and (b) 
nature of the temporal evolutions, e.g. modifications in the shape of the objects are more frequent in the Lower Aude Valley since the site is exposed to flooding events. In addition, the fact of having many small parcels (near to the limit of detection) may contribute to shape instability from a timestamp to the next one.

\subsection{Spatiotemporal Dynamics}

When repeatability and compatibility of satellite observations are guaranteed it becomes possible to detect spatiotemporal evolutions, from which the related dynamics can be deduced (Bonn, 1996). In that light, we consider spatiotemporal dynamics as derived from a set of consecutive evolutions we detected throughout the time series. In particular, we performed analysis at both graph and study-site levels (as described in Section 2.5).

\subsubsection{Graph Level Analysis}

In order to better illustrate graph structure and content, we selected 4 graphs representing different evolutions in time (see Figure 7). Graph A represents a natural area composed mainly by scrubland and forest. Its $B B$ came from the fourth timestamp which corresponds to the beginning of the summer. At this time, the area is the most homogeneous while the most heterogeneous situations are observed in the first (winter) and third (spring) timestamps. In those two timestamps it is possible to better distinguish the deciduous vegetal community (brown at $T_{1}$ and light green at $T_{3}$ ) from the surrounding coniferous community (dark green during the whole time series). Conversely, Graph B has a particular structure with two very distinct portions: first there is a single object per timestamp from $T_{0}$ to $T_{3}$ whereas from $T_{4}$ to $T_{5}$ there are several objects per timestamp ( 8 and 6 respectively). The huge spatial fractioning observed between $T_{3}$ and $T_{4}$ corresponds to the drying-up process of the Pisse-vaches coastal lagoon. High evaporation rates combined to weak precipitations during the summer leads to the replacement of the lagoon by a wide dry salt flat in the end of this season. Graph C presents a quite similar, but inverted, structure w.r.t. Graph B. In fact, this saltmarsh and salt meadow area is more heterogeneous in the beginning of the time series $\left(T_{0}\right.$ up to $\left.T_{2}\right)$. At this time, the area is partially covered by water and hygrophilous vegetation, which explains the dissimilarities on the objects boundaries during these three timestamps. Afterwards, water is no more present and the dry summer conditions lead to a fast decrease of the photosynthetic vegetation, as a consequence, the area becomes much more homogeneous in the three last timestamps. Finally, Graph D represents the evolutions over two adjacent cereal crop fields. The plant-growing season is visible from $T_{0}$ to $T_{2}$ (late winter to spring) although we can notice that plant-growing is not homogeneous all over the field area. Then, the crops are harvested in early summer (between $T_{2}$ and $T_{3}$ ) and both fields remain unvegetated until the end of the time series.

In addition to graph structure and visual analysis of image objects, it is important to consider the changes in the content of the objects. For that purpose, each graph can be also finely analyzed thanks to Temporal Profiles representing the variation of any object attribute. As examples, we can use the previously discussed graphs B and D (see Figure 8). In the case of Graph B, the temporal behavior of VSDI furnishes reliable information about the drying-up process of the coastal lagoon as this spectral index is sensitive to changes in soil 


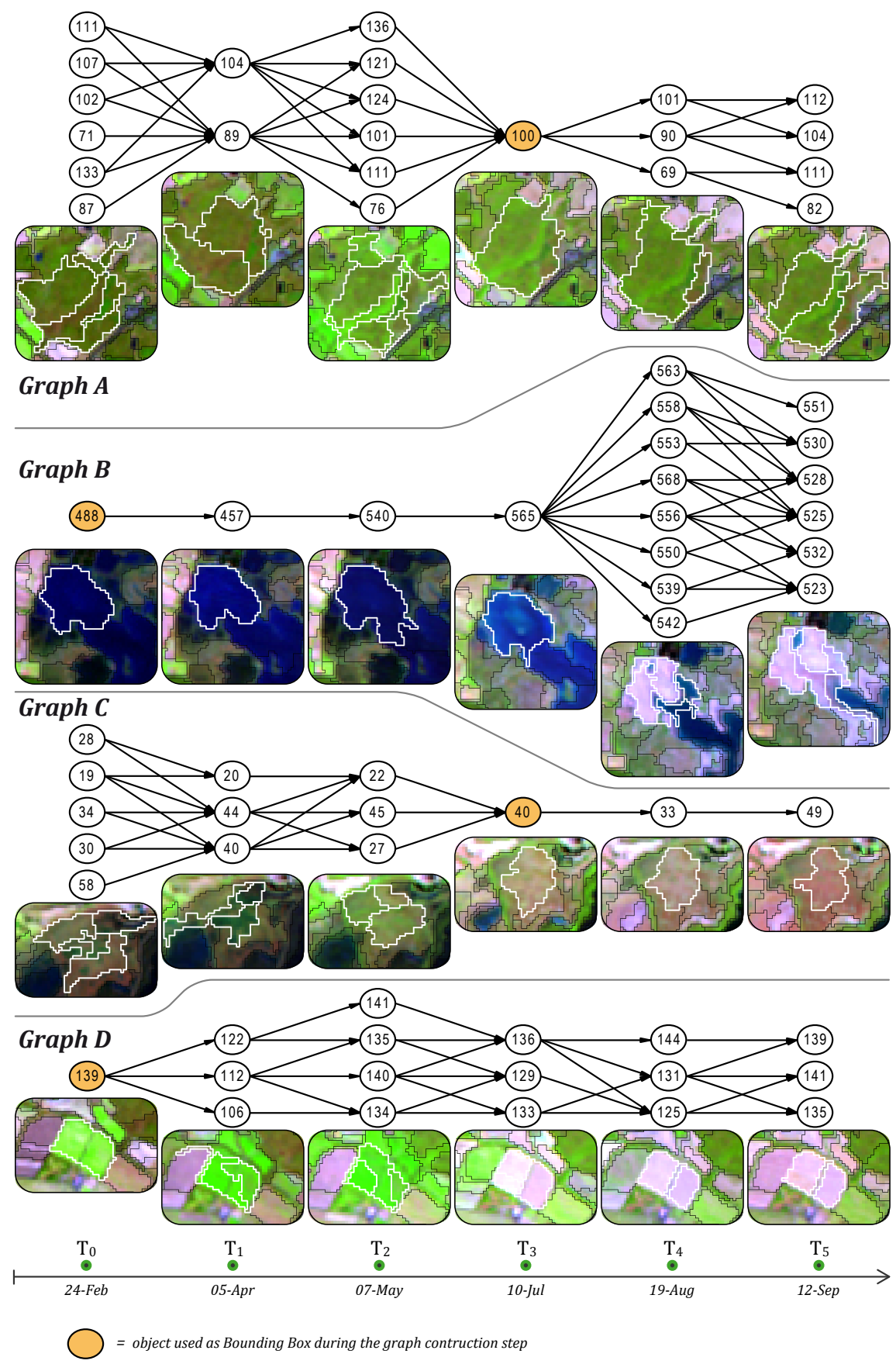

Figure 7: Examples of graphs showing different structures and representing distinct evolutions in time. A- a scrubland and forest area (central part of the Libron site), B- a coastal lagoon (southern part of the Lower Aude Valley), C- a saltmarsh area (northern part of the Lower Aude Valley), D- two crop fields (near to the central part of the Libron site). 

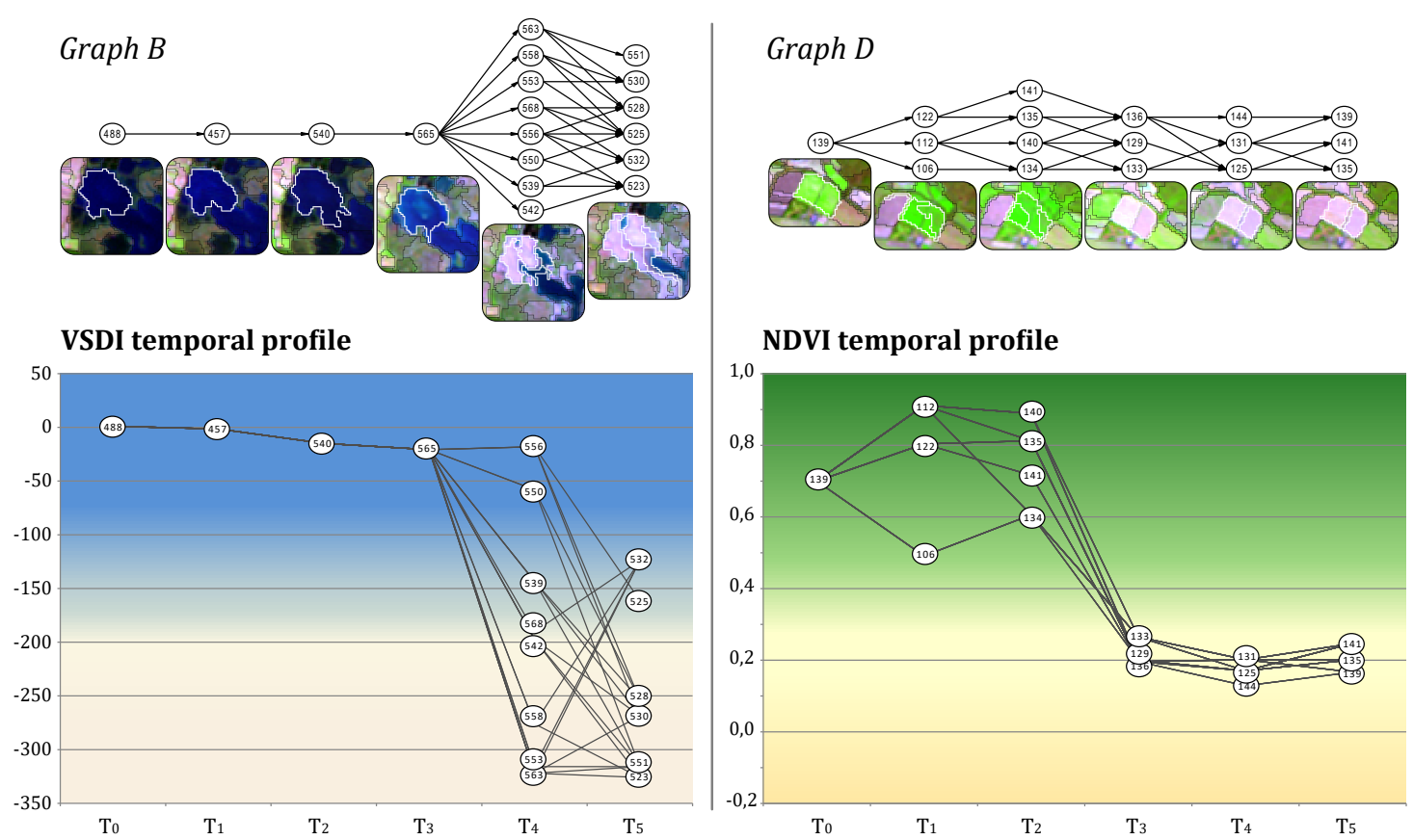

Figure 8: Temporal profiles for two selected graphs (see 7). For Graph B (left) the temporal profile corresponds to the variation of the VSDI (each object is represented by its mean VSDI value) while for Graph D (right) it corresponds to the variation of the NDVI (each object is represented by its mean NDVI value).

moisture and to the presence of surface water. Likewise, for Graph D, the NDVI temporal profile is useful to follow the changes observed over the cereal crop fields, i.e. plant-growing, harvest and long-lasting bare soil.

\subsubsection{Study-site Level Analysis}

Beside such fine temporal information, the Global Variation (GlobalVar) synthesizes how much the area represented by a given graph evolves during the whole time series and a GlobalVar map can be built from this information. Several GlobalVar maps can be produced by combining different object attributes. Indeed, GlobalVar maps are useful to compare graphs and promptly detect the most and the less stable areas within the considered study sites. In our case, as both study sites have the same timestamps, GlobalVar maps can also be used to compare the two zones (see Figure 9).

Regarding Figure 9, we can observe that choosing different attribute combinations results in somewhat different GlobalVar maps. We can also underline that the two study sites exhibit different behaviors considering different attribute combinations. In other words, the attributes showed in Figure 9 are differently correlated according to each site and, in general, they are not highly correlated among them. Regardless of the attribute selection, the Libron site presents invariably higher values of GlobalVar if compared to the Lower Aude Valley. This is more evident when only the NDVI is employed (map 1) or only the raw bands (map 2) to produce maps by means of GlobalVar, instead of map (3) where all the spectral indices were used. 

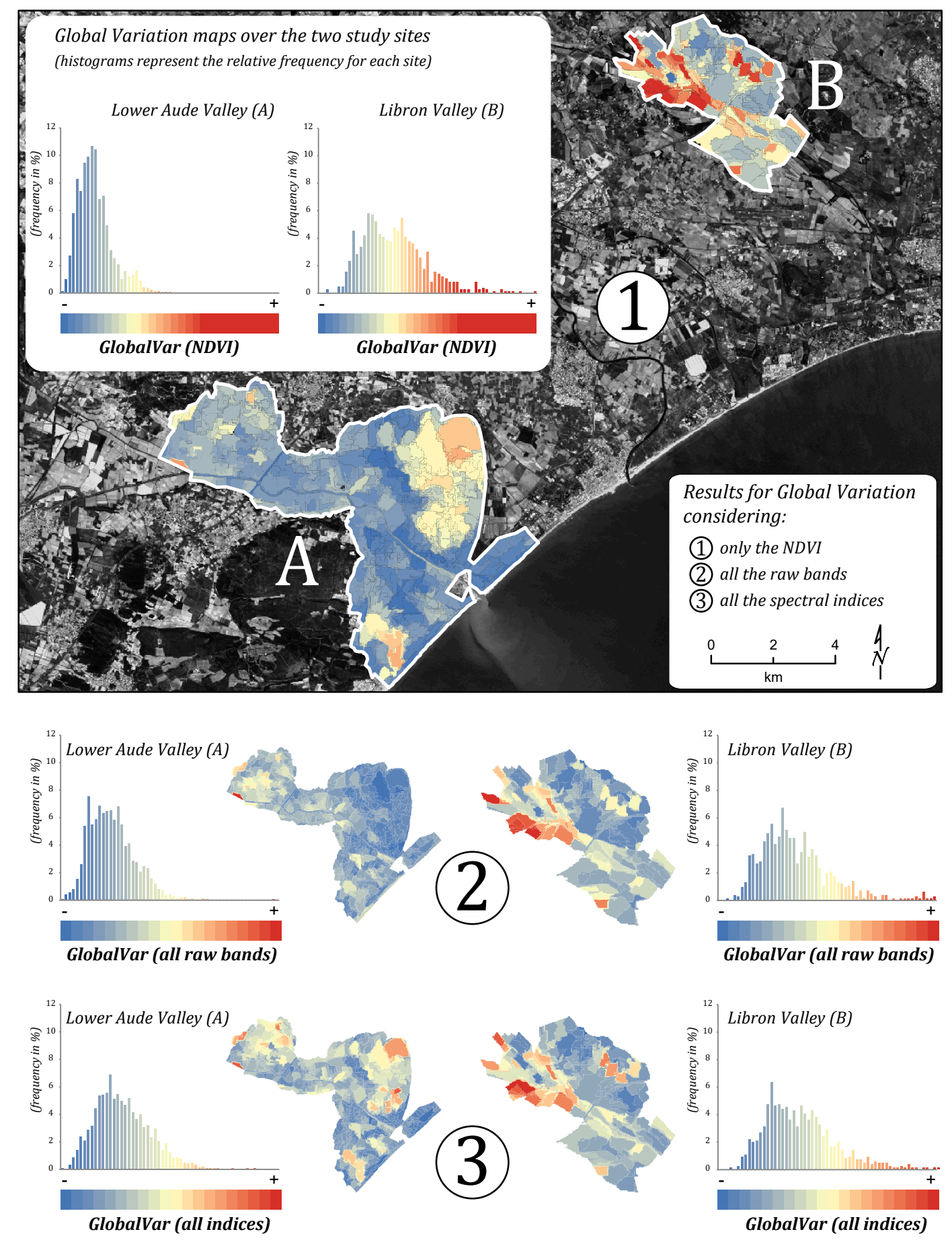

Figure 9: Global Variation (GlobalVar) maps and the corresponding frequency histograms for the two study sites (A-Lower Aude Valley, B-Libron Valley). The following attributes were used for the GlobalVar maps: (1) only the NDVI, (2) all the raw bands and (3) all the spectral indices. As graph coverages may partially overlap, the GlobalVar values has been recalculated, for all the overlapping areas, considering the proportional contribution of the involved graphs. 
Considering only the Lower Aude Valley, one can suppose a very stable situation based on the analysis of the second map (all raw bands). Excepting few cereal crops in the western part of the site, all the remaining areas present low values of GlobalVar, including all the temporary flooded areas. Alone, the six spectral bands provide a very partial representation of the temporal dynamics since only some radical evolutions (i.e. bare soil/dense vegetation/bare soil) are highlighted, while the other evolutions are not took into account. Over the Libron Valley site such kind of radical evolution is more frequent and widespread, especially in the crop areas located along the upstream Libron waterway (red and orange hues in map 2). However, even in this case the results are not satisfactory as some crops areas presenting radical evolutions displays medium values of GlobalVar instead of high values.

Conversely, the GlobalVar map derived from the NDVI furnishes much more reliable information related to landscape dynamics in both study sites. In the Libron Valley, the highest values correspond to the agricultural areas where important changes are observed throughout the year (e.g. cereals, sunflower, flower-fruit vegetables and seeds). Vineyards and orchards generally experience less noticeable inter-annual variations and are therefore assigned with medium or medium-low GlobalVar values. The lowest values correspond mostly to natural scrub and forest areas, in particular those dominated by coniferous. In the Lower Aude Valley, the lowest values are also principally related to natural areas, more specifically to herbaceous/scrub vegetation covering sand dunes (southeast of the site) or some not submerged areas surrounding the Vendres lagoon (center of the site). In addition, some build-up areas like camping and recreational facilities (all located along to the coast) present low GlobalVar values. Vineyards and orchards, as well as saltmarsh and salt meadow areas, are generally assigned with medium or medium-low GlobalVar values. Finally, the most dynamic areas are clearly associated to the two coastal lagoons (Vendres and Pissevaches) and, in a minor extent, to the few cereal crops located in the western part of the site. The graphs representing the coastal lagoons and surrounding areas are characterized by important changes in the objects shape and content. The Pisse-vaches sector is temporarily covered by shallow and brackish waters. It is scarcely colonized by the vegetation either during the submerged periods (very few aquatic macrophyte during winter and spring) either during the waterless period (very limited growth of pioneer communities during the summer and autumn). The Vendres sector also presents important seasonal water level fluctuations but possess a permanently flooded area (northeastern portion). Salinity is less important in this sector where dense aquatic and terrestrial vegetation can be observed during spring and summer.

Finally, the third map of Figure 9 combines the three spectral indices to compute the GlobalVar (all indices). The spatial distribution of the less and the most dynamic areas is somehow similar to those described for the NDVI GlobalVar map, which is logical as the NDVI is one of the three spectral indices considered here. Nevertheless, the inclusion of VSDI and NDWI draw attention to some temporal evolutions unnoticed by the NDVI, in particular over the areas where the contribution of the soil is greater than those of the vegetation. 


\begin{tabular}{lcc}
\hline & Pearson's $r$ & Spearman's rho \\
\hline All raw bands & 0.574 & 0.476 \\
NDVI & 0.767 & 0.659 \\
NDWI & 0.787 & 0.672 \\
VSDI & 0.719 & 0.580 \\
All indices & 0.789 & 0.676 \\
\hline
\end{tabular}

Table 4: Correlation coefficients results for ancillary based verification. The value of change from ancillary imagery (VCA) was compared with five sets of GlobalVar (all raw bands, NDVI, NDWI, VSDI, all indices)

\subsection{Ancillary imagery based verification}

As explained in section 3.3.1, the ancillary imagery was processed in order to estimate the intensities of change all over the Lower Aude Valley Natura 2000 site. Such intensities of change were obtained by comparing two land cover maps and their related from-to evolution classes. The first map (derived from aerial photographs) represented the study site in May 2009, while the second one (based on a RapidEye image) represented the site in late June 2009. This time interval corresponds roughly to the timestamps $T_{2}$ (7 May 2009) and $T_{3}$ (10 July 2009) of our Landsat time series. To perform a coherent verification, we calculated an extra set the GlobalVar values considering only these two timestamps of the former time series.

As the spatial boundaries between the evolution graphs and the land cover maps are not similar, we employed the following strategy to compare their intensities of change. Starting from the CoreCov of each graph, we clipped the corresponding polygon(s) of the land cover maps. If a given graph is represented by more than one map polygon, we computed a weighted average of the intensities of change of those polygons. This is done by taking into account the relative area of each map polygon (inside the CoreCov) and multiplying it by a coefficient of change. The coefficient varies according to the intensities of change (low $=1$, medium $=2$, high $=3$ and very high=4) assigned to the from-to evolution classes. As consequence, each graph received a new numerical value of change (VCA) that is derived from the ancillary imagery and can be therefore compared to the GlobalVar values.

Table 4 summarizes the correlation coefficients obtained from the comparison of VCA against five sets of GlobalV ar (all raw bands, NDVI, NDWI, VSDI, all indices). We used two coefficients of correlation: (a) the Pearsons coefficient which measures the strength of the association between two variables and (b) the Spearmans ranked coefficient which assumes that the two variables under consideration were measured on an ordinal scale.

The strength of association is particularly high between VCA and three sets of GlobalVar (all indices, NDWI and NDVI). This assertion is valid for the two correlation methods: (a) Pearson's r ranging from 0.767 to 0.789 and (b) Spearman's rho ranging from 0.659 to 0.676. For both Pearson and Spearman, the correlation coefficient is very highly significantly different from zero ( $\mathrm{p}$-value $<0.0001$ ).

It is worth noting that we eliminated the areas with potential water level fluctuations from this comparison. This was necessary because the acquisition dates of the images (Landsat and ancillary imagery) are not the same (i.e. 15 days separates the RapidEye 
image from its corresponding Landsat image). As the water level is highly variable around the two coastal lagoons, we cannot assume that the observations made with several days of interval are comparable. During this time interval, other short-time evolutions (such as crop harvesting or plant growing) can occur and dramatically change the observed landscape. However, the detection of all these not comparable areas over the entire study site would require a very careful and meticulous visual confrontation of the 4 images employed in the verification process. Even without performing such deep data-cleaning task, we obtained high correlations between VCA and most sets of GlobalVar values. As expected, the strength of association was stronger when using spectral indices instead of raw bands. The best correlation coefficient was obtained with the GlobalVar(all indices), which considers the behavior of the three spectral indices together. Individually, both the GlobalVar(NDVI) and the GlobalVar(NDWI) are highly correlated to VCA. Although the difference is small, the combination of the three indices furnished an automated evaluation of the evolutions that is the nearest of those obtained from manual digitalization and visual interpretation of the ancillary images.

\subsection{Thematic layer based verification (official farmer declarations)}

As the boundaries of the thematic layer are not similar to those of the evolution graphs, we employed the following strategy. Starting from the 32 polygons representing the declared groups of culture by the farmers in 2009, we clipped the corresponding evolution graphs representing such areas (the CoreCov of each graph). If a given polygon of culture is represented by more than one evolution graph, we computed a weighted average of the GlobarVar values of those graphs. This is done by taking into account the relative area of each graph (inside the polygon of culture) and multiplying it by the corresponding Globar Var values. At the end, each polygon of culture was assigned with five values of GlobalVar (all raw bands, NDVI, NDWI, VSDI, all indices) derived from the evolution graphs representing such agricultural areas.

Figure 10 summarizes, for each group of culture, the mean GlobalVar value obtained for the five sets of attributes (all bands, NDVI, NDWI, VSDI, all indices). The number of polygons available for each group of culture is indicated in Figure 10 as well as the error bars related to the mean values (excepted for the sunflower crop that possesses only a single polygon).

The general analysis of Figure 10 allows grouping the cultures into two subsets: (a) vineyard and orchard which presented low GlobalVar values and (b) cereals (excepted wheat), sunflower, seeds and flower-fruit vegetables which presented high GlobalVar values. This separation in two main subsets can be observed in any of the five plots but is most evident in GlobalVar (NDVI).

Although the number of polygons is quite small (32), the results are consistent with the dynamics we can observe for those types of culture. Orchards and vineyards parcels present almost the same temporal evolutions with a gradual augmentation of the greenness during spring and early summer. Then, the greenness level remains nearly stable for most of the parcels while for some others it can decrease thinly up to late summer. One interesting difference between these cultures is that the maximum of greenness is attained firstly for 

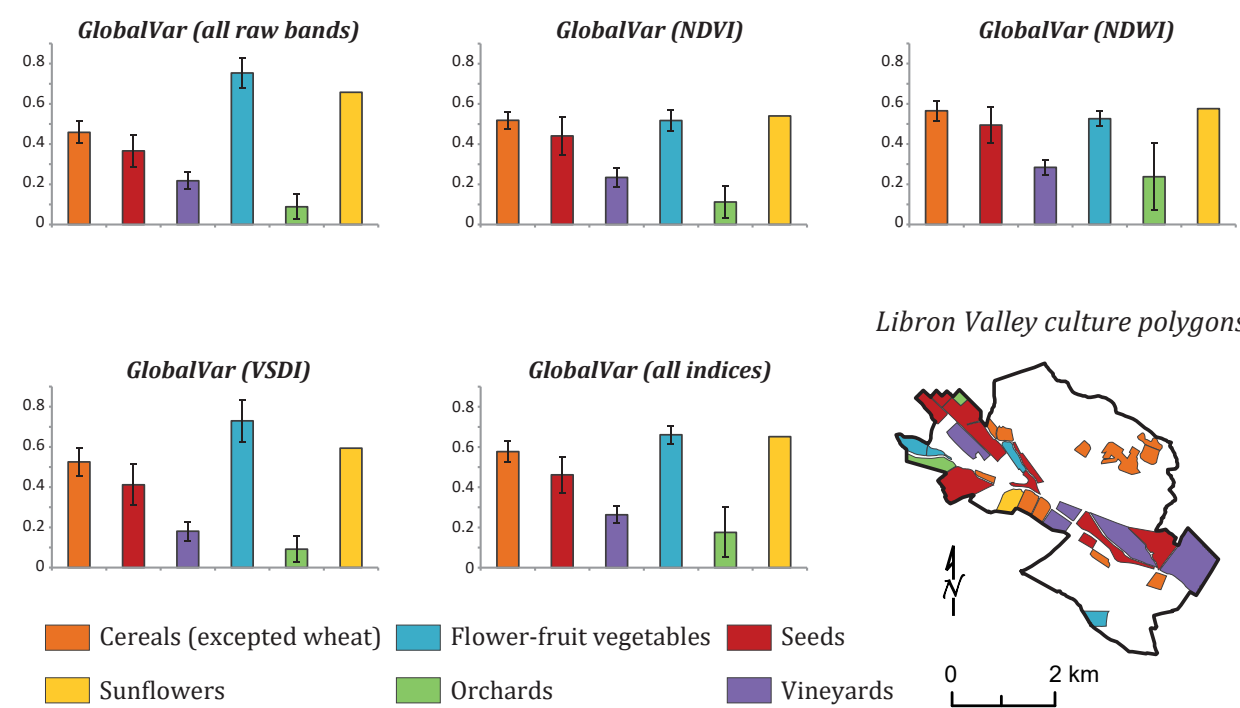

Libron Valley culture polygons
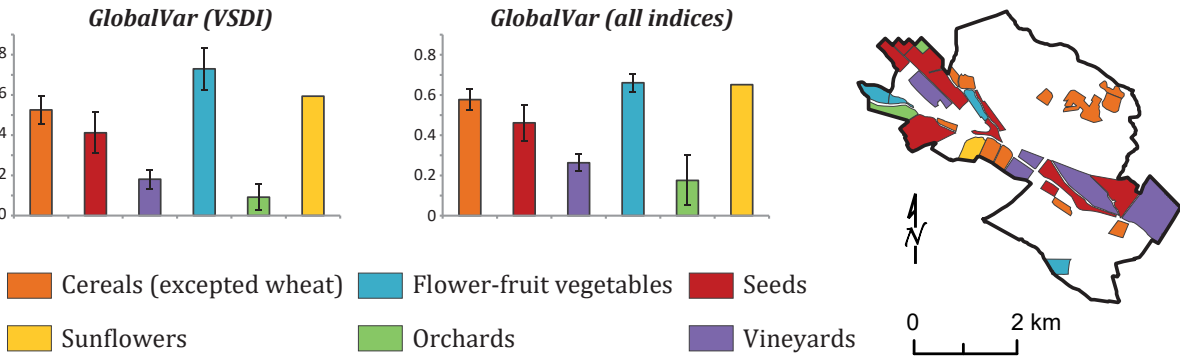

Figure 10: Thematic layer verification using official farmer declarations.

the orchards (usually at $T_{2}$ in our time series) and lately for the vineyards (usually at $T_{3}$ in our time series). These may explain why the mean values of GlobalVar are smaller for the orchards if compared with those of the vineyards. The second subset assembles four types of crops: cereals, sunflower, seeds and flower-fruit vegetables. Those crops present dissimilar temporal dynamics but all of them are composed by the same general evolutions, or phases: plant-growing, harvest and bare soil. According to the calendar of each crop, such phases are observed in different periods of the time series. In a simplified way, they are temporally distributed as follows: (i) between $T_{0}$ and $T_{2}$ plant-growing for cereals and bare soil for the other cultures, (ii) between $T_{2}$ and $T_{3}$ harvesting for cereals and plant-growing for the other cultures, (iii) between $T_{3}$ and $T_{4}$ bare soil for cereals, harvesting for flower-fruit vegetables and plant-growing for sunflower and seeds, (iv) between $T_{4}$ and $T_{5}$ bare soil for cereals and flower-fruit vegetables, harvesting for sunflower and seeds. As we can observe, the evolutions are shifted in time, but their corresponding length over the entire time series is very close. As a consequence, the GlobalVar calculated for these crops generally fit in the same range of values (taking into account the error bars).

According to the results of the two independent verifications presented in this section, our framework produced reliable results that are consistent with the ground truth we employed. More in detail, we have seen that our methodology is able to automatically detect spatiotemporal dynamics in natural, semi-natural and agricultural areas.

The object-tracking mechanism we conceived is able to describe those dynamics by means of objects coming from different images of the time series. Once the graph structure is built, it can be analyzed considering different combinations of the object attributes (e.g. raw bands, spectral indices). Modifying the combination of object attributes introduces some kind of flexibility and it allows to customize the proposed framework according to the task the user wants to deal with. 
The proposed framework can be also exploited in order to plan field campaigns on unknown areas since it supplies an exploratory tool to draw a global overview of a study area. The practical interest is twofold: firstly, it provides a synoptic view based on spatially coherent areas over the time; secondly, for each of these areas is automatically characterized by an estimation how much it evolved during the whole period covered by the SITS.

\section{Conclusion}

In this paper we proposed a new methodological framework to automatically extract spatiotemporal information from SITS. We combined OBIA and data mining techniques to extract graph structures describing spatio-temporal dynamics from SITS. Our approach starts with classical OBIA image processing which results in separate sets of objects for each timestamp. Then, a graph-based approach is employed to detect spatially coherent areas and connect the objects belonging to different timestamps generating a set of evolution graphs. From these graphs, spatio-temporal dynamics are computed and summarize the temporal behavior of each particular area over the time.

The proposed framework was evaluated in two study sites located in the South of France, near to the Mediterranean Sea. The experiments underlined how the extracted information can be deeply explored at the evolution graph scale, as well as to supply a general picture at the study site scale, but also to be used for comparing different study sites. The robustness of the framework is verified via ancillary imagery, field campaigns and official farmer declarations.

The framework described in this paper can be potentially used with any kind of SITS. In particular, Sentinel-2 images will shortly improve the observational capabilities for monitoring purposes (the first satellite started operational acquisition in the beginning of 2016). Enhanced revisit capacity, i.e. 5 days when the two satellites will become operational, associated to $10 \mathrm{~m}$ resolution for visible and NIR bands, will open new possibilities for several applications including the monitoring of natural and agricultural areas.

As a future work we plan to exploit deeply the knowledge supplied by the graph-based representation. One of our ongoing works is related to automatically grouping similar spatiotemporal entities in order to define categories (or families) of evolutions that characterize a given study site.

\section{Acknowledgment}

The authors would like to acknowledge the French National Research Agency in the framework of the program "Investissements d'Avenir" (GEOSUD project, ANR-10-EQPX20) and the National French Center for Spatial Study (CNES) in the framework of the project "DYNAMITEF TOSCA 2016".

\section{References}

Achanta, R., Shaji, A., Smith, K., Lucchi, A., Fua, P., Süsstrunk, S., 2012. SLIC superpixels compared to state-of-the-art superpixel methods. IEEE Trans. Pattern Anal. Mach. Intell. 34 (11), 2274-2282. 
B. Descle, P. Bogaert, P. D., 2006. Forest change detection by statistical object-based method. Remote Sensing of Environment 102 (12), 1 - 11.

Baatz, M., Hoffmann, C., Willhauck, G., 2008. Progressing from object-based to object-oriented image analysis. Lecture Notes in Geoinformation and Cartography. Springer Berlin Heidelberg, Ch. 2, pp. 2942.

Blaschke, T., 2005. Towards a framework for change detection based on image objects. Göttinger Geographische Abhandlungen 113, 1-9.

Blaschke, T., 2010. Object based image analysis for remote sensing. ISPRS Journal of Photogrammetry and Remote Sensing 65 (1), 2-16.

Blaschke, T., Hay, G. J., Kelly, M., Lang, S., Hofmann, P., Addink, E., Queiroz Feitosa, R., van der Meer, F., van der Werff, H., van Coillie, F., Tiede, D., 2014. Geographic object-based image analysis towards a new paradigm. ISPRS Journal of Photogrammetry and Remote Sensing 87 (0), 180-191.

Bonn, F., 1996. Précis de télédétection: Applications thématiques. Vol. 2. Sillery: Presses de l'Université du Québec.

Cai, S., Liu, D., 2015. Detecting change dates from dense satellite time series using a sub-annual change detection algorithm. Remote Sensing 7 (7), 8705.

Chen, G., Hay, G. J., Carvalho, L. M. T., Wulder, M. A., 2012. Object-based change detection. International Journal of Remote Sensing 33 (14), 4434-4457.

Coppin, P., Jonckheere, I., Nackaerts, K., Muys, B., Lambin, B., 2004. Digital change detection methods in ecosystem monitoring: a review. International Journal of Remote Sensing 25 (9), 1565-1596.

Gao, B.-C., 1996. Ndwi a normalized difference water index for remote sensing of vegetation liquid water from space. Remote Sensing of Environment 58 (3), 257-266.

Hagolle, O., Huc, M., Pascual, D. V., Dedieu, G., 2010. A multi-temporal method for cloud detection, applied to formosat-2, vens, landsat and sentinel-2 images. Remote Sensing of Environment 114 (8), 1747-1755.

Hussain, M., Chen, D., Cheng, A., Wei, H., Stanley, D., 2013. Change detection from remotely sensed images: From pixel-based to object-based approaches. ISPRS Journal of Photogrammetry and Remote Sensing 80 (0), 91-106.

Inglada, J., Arias, M., Tardy, B., Hagolle, O., Valero, S., Morin, D., Dedieu, G., Sepulcre, G., Bontemps, S., Defourny, P., Koetz, B., 2015. Assessment of an operational system for crop type map production using high temporal and spatial resolution satellite optical imagery. Remote Sensing 7 (9).

Jackson, T. J., Chen, D., Cosh, M., Li, F., Anderson, M., Walthall, C., Doriaswamy, P., Hunt, E. R., 2004. Vegetation water content mapping using landsat data derived normalized difference water index for corn and soybeans. Remote Sensing of Environment 92 (4), 475-482.

Lillesand, T. M., Kiefer, R. W., Chipman, J. W., 2008. Remote Sensing and image interpretation. John Wiley \& Sons.

Lu, D., Mausel, P., Batistella, M., Moran, E., 2005. Landcover binary change detection methods for use in the moist tropical region of the amazon: a comparative study. International Journal of Remote Sensing 26 (1), 101-114.

Lunetta, R. S., Knight, J. F., Ediriwickrema, J., Lyon, J. G., Worthy, L. D., 2006. Land-cover change detection using multi-temporal \{MODIS\} \{NDVI\} data. Remote Sensing of Environment 105 (2), 142 154.

Malila, W. A., 1980. Change vector analysis: an approach for detecting forest changes with landsat. In: LARS Symposia. p. 385.

Maurer, S. B., 2003. Directed Acyclic Graphs. CRC press.

Nagendra, H., Lucas, R., Honrado, J. P., Jongman, R. H., Tarantino, C., Adamo, M., Mairota, P., 2013. Remote sensing for conservation monitoring: Assessing protected areas, habitat extent, habitat condition, species diversity, and threats. Ecological Indicators 33, 45-59.

Petitjean, F., Kurtz, C., Passat, N., Ganarski, P., 2012. Spatio-temporal reasoning for the classification of satellite image time series. Pattern Recognition Letters 33 (13), 1805-1815.

Qin, Y., Niu, Z., Chen, F., Li, B., Ban, Y., 2013. Object-based land cover change detection for cross-sensor images. International Journal of Remote Sensing 34 (19), 6723-6737. 
Rouse Jr, J., Haas, R., Schell, J., Deering, D., 1974. Monitoring vegetation systems in the great plains with erts. NASA special publication 351, 309

Sagris, V., Devos, W., 2008. Lpis core conceptual model: methodology for feature catalogue and application schema. Tech. rep., Joint Reaearch Centre of European Commission: Ispra, Italy.

Singh, A., 1989. Review article digital change detection techniques using remotely-sensed data. International Journal of Remote Sensing 10 (6), 989-1003.

Tucker, C. J., 1979. Red and photographic infrared linear combinations for monitoring vegetation. Remote Sensing of Environment 8 (2), 127-150.

Vanden Borre, J., Paelinckx, D., Mcher, C. A., Kooistra, L., Haest, B., De Blust, G., Schmidt, A. M., 2011. Integrating remote sensing in natura 2000 habitat monitoring: Prospects on the way forward. Journal for Nature Conservation 19 (2), 116-125.

Verbesselt, J., Hyndman, R., Newnham, G., Culvenor, D., 2010. Detecting trend and seasonal changes in satellite image time series. Remote sensing of Environment 114 (1), 106-115.

West, D. B., 2001. Introduction to graph theory, 2nd Edition. Prentice-Hall.

Yuan, F., Sawaya, K. E., Loeffelholz, B. C., Bauer, M. E., 2005. Land cover classification and change analysis of the twin cities (minnesota) metropolitan area by multitemporal landsat remote sensing. Remote Sensing of Environment 98 (23), 317-328.

Zhang, N., Hong, Y., Qin, Q., Liu, L., Zhang, N., Hong, Y., Qin, Q., Liu, L., 2013. Vsdi: a visible and shortwave infrared drought index for monitoring soil and vegetation moisture based on optical remote sensing. International Journal of Remote Sensing 34 (13), 4585-4609. 